\title{
Presentación de un caso de un recién nacido prematuro con COVID-19.
}

Case report of a premature newborn with COVID-19.

\author{
Beatriz Gaytán-Flores ${ }^{a}$, Aldair Hernández-Moreno ${ }^{b}$, Reyna Cristina Jimenez Sánchez ${ }^{c}$, Rosario \\ Barrera-Galvez, Jose Arias-Rico ${ }^{e}$
}

\begin{abstract}
:
Pregnant women are a susceptible population for SARS-CoV-19 and are more likely to have complications and even progress to severe disease. The aim of this study is to present the case of a neonatal patient (preterm 34 SDG) with COVID19. This is a descriptive study, in which we report a case of neonatal COVID-19 (neonate 34 SDG) in the third-level hospital of the State of Mexico. The case of a newborn with 34 SDG positive for SARS Cov-2 is presented. This document describes the clinical evolution from admission to discharge, with a favorable evolution. Clinical manifestations, especially in preterm patients, are unspecific; therefore, it is necessary to carry out a specific check-up such as monitoring of vital signs. Therefore, the patient who is a child of a symptomatic covid-19 positive mother, who is also premature, requires follow-up and surveillance in the NICU. It is fundamental and crucial to know in depth the optimal conduction in the neonatal patient diagnosed with SARS CoV-2, the study shows that following current protocols and scientific evidence has a positive impact in the hospital stay and complications tend to decrease
\end{abstract}

Keywords:

COVID-19; SARS COV-2; Neonate; coronavirus in neonates; case report

Resumen:

Las mujeres embarazadas son una población susceptible de COVID-19 y son más propensas a tener complicaciones e incluso progresan hasta convertirse en una enfermedad. grave. El objetivo de este estudio es presentar el caso de un paciente neonatal (prematuro de 34 SDG) con COVID19. Se trata de un estudio descriptivo, en el que informamos de un caso de COVID-19 neonatal (neonato 34 SDG) en un hospital de tercer nivel del Estado de México. Se presenta el caso de un recién nacido de 34 SDG positivo a SARS Cov-2, en este documento se describe la evolución clínica desde su ingreso hasta su egreso, con una evolución favorable. Las manifestaciones clínicas, sobre todo en pacientes pretérmino, son inespecíficas; por lo tanto es necesario llevar a cabo una revisión específica como monitorización de los signos vitales y una. Por lo tanto el paciente hijo de madre positiva a covid-19 sintomático que además es prematuro, requiere seguimiento y vigilancia en la UCIN. Es fundamental y crucial conocer a fondo la conducción óptima en el paciente neonatal diagnosticado con SARS CoV-2, en el estudio se demuestra que el seguir protocolos actuales y evidencia científica impacta de manera positiva ya que la estancia hospitalaria y complicaciones tienden a disminuir.

\section{Palabras Clave:}

COVID-19, SARS COV-2, Neonato, coronavirus en neonatos, reporte de caso.

\footnotetext{
a Beatriz Gaytan-Flores https://orcid.org/0000-0002-9707-633X Email: ga232994@uaeh.edu.mx

${ }^{\mathrm{b}}$ Aldair Hernandez-Moreno https://orcid.org/0000-0003-0059-5828, Email: aldahir_hernandez@uaeh.edu.mx

• Reyna Cristina Jiménez-Sánchez; Universidad Autónoma del Estado de Hidalgo, https://orcid.org/0000-0001-9264-8514, Email: cristiji@hotmail.com

d Rosario Barrera-Gálvez; Universidad Autónoma del Estado de Hidalgo, https://orcid.org/0000-0002-1949-5424, Email: rosario_barrera@uaeh.edu.mx

e Autor de Correspondencia; José Arias-Rico Universidad Autónoma del Estado de Hidalgo, https://orcid.org/0000-0003-0219-0410, Email: jose_arias@uaeh.edu.mx
} 


\section{Introducción}

En diciembre de 2019, una neumonía causada por el nuevo síndrome agudo respiratorio coronavirus-2 (SARS-CoV-2) surgió en Wuhan, provincia de Hubei, China. Desde 2019 la enfermedad coronavirus (COVID-19) es altamente contagiosa con una tasa alta de mortalidad, fue clasificada como una enfermedad infecciosa de clase B. ${ }^{1,2}$ En los últimos 20 años, el SARS-CoV y el MERS-CoV ha infectado > 10.000 personas en todo el mundo. La tasa de mortalidad de la infección por el SARS-CoV es del $10 \%$, la tasa de infección por el SARS-CoV en las mujeres embarazadas es del $25 \%$, y la tasa de mortalidad de la infección por el MERS-CoV es de hasta un 37\%. Las mujeres embarazadas son una población susceptible al SARSCoV-2 y es más probable que tengan complicaciones e incluso que progresen a una enfermedad grave. ${ }^{3}$

Ciertas revisiones retrospectivas en mujeres embarazadas con COVID-19 no evidenciaron infección intrauterina. Sin embargo, no se puede descartar la transmisión vertical. De los datos de infecciones por otros coronavirus, como el síndrome respiratorio agudo grave (SARS), que afectó a más de 8,400 Personas en el mundo, se estimó un compromiso aproximado de 100 gestantes, con una tasa de letalidad hasta del $30 \%$, con peores desenlaces al compararlas con mujeres no gestantes y complicaciones adicionales dadas por abortos espontáneos y restricción del crecimiento intrauterino. ${ }^{2}$

Un pequeño número de casos con la enfermedad por coronavirus 2019 (COVID-19, por sus siglas en inglés) ha sido descrito hasta la fecha en niños y, en comparación con los casos diagnosticados en adultos, tiene un espectro clínico limitado y hasta el momento los datos aportados por estudios realizados en China y otros países refieren que la evolución de la enfermedad es menos severa en los infantes en relación a los adultos. ${ }^{5}$

En los casos declarados por el centro chino para el control y la prevención de enfermedades, sólo el $2 \%$ de los casos tenían entre 0-19 años. Los pacientes pediátricos presentan un mejor pronóstico mostrando un periodo de recuperación de 1-2 semanas, pero con síntomas leves. Los autores indican que esto no significa que los niños no sean vulnerables, sino que no suelen presentar síntomas severos. Por otra parte, existen datos de los registros de la infección por SARS-CoV-2 que indican que la incidencia en la edad pediátrica va en aumento. ${ }^{6}$

Hasta febrero del 2020, el $2.4 \%$ de los 75,465 casos (confirmados y sospechosos) en China se reportaron en la población pediátrica. Mientras que, en Estados Unidos, al 2 de abril 2020 del total de casos 149,082, 1.7\% (2,572) corresponden a casos pediátricos (menores 18 años) y dentro de este grupo la mayoría son menores de 1 año (0.3\%). En este grupo norteamericano de niños, se reporta un porcentaje de hospitalización del 5-10\% y un ingreso a Unidad de Cuidados Intensivos (UCI) del 0.5-2\%. ${ }^{7}$

\section{Materiales y Métodos}

Este es un trabajo descriptivo de estudio de caso, en el que se presenta un paciente con una patología no muy común y de actualidad, pues es prematuro con COVID-19. Se describe la evolución del caso y cómo fue que este paciente llegó a dar positivo para dicha patología.

\section{Definición operacional.}

El INPER (2020) considera caso sospechoso al recién nacido asintomático o con enfermedad respiratoria aguda leve o grave, fiebre o tos que tenga alguna de las siguientes características:

a. Ser hijo de madre con enfermedad respiratoria aguda leve o grave que sea caso confirmado o bajo investigación a COVID-19 desde 14 días antes, al momento del nacimiento o durante los primeros 28 días de vida.

b. Haber estado en contacto con cualquier persona con enfermedad respiratoria aguda leve o grave que sea caso confirmado o bajo investigación COVID-19 durante los primeros 28 días de vida.

\section{Caso confirmado.}

Todos los recién nacidos de mujeres con confirmación de COVID-19 y que presentan síntomas de infección se les debe descartar con pruebas de laboratorio COVID-19. La separación preventiva de rutina de una madre y un bebé sano no debe realizarse a la ligera, debido a los posibles efectos perjudiciales en la alimentación y la vinculación. ${ }^{8}$

\section{Cuadro clínico neonatal.}

El espectro clínico de infección neonatal por COVID es inespecífico. Puede cursar asintomática o con dificultad respiratoria leve a severa, tos, fiebre, cianosis, datos de respuesta inflamatoria sistémica, compromiso cardiovascular y se han descrito biometría hemática normal o con leucopenia o linfopenia. Otros hallazgos como trombocitopenia y/o elevación de enzimas hepáticas, puede acompañarse de datos radiológicos sugestivos de neumonía y en otras ocasiones de íleo séptico.

\section{Resultados}

Presentación del caso.

En julio del 2020 ingresa madre adolescente primigesta de 17 años con datos francos de Eclampsia y ruptura prematura de membranas de aproximadamente 48 horas, por lo que se realizó una cesárea urgente induciendo sedación y analgesia a la semana 32 por ultrasonido. Cuatro horas posteriores al procedimiento se realiza tomografía de tórax por protocolo de la institución donde se observan imágenes sugestivas de neumonía con alta sospecha de COVID-19, por lo que se solicita una técnica de reacción en cadena de polimerasa de transcripción inversa en tiempo real del coronavirus 2019 (RT- 
PCR COVID-19), la cual se reportó POSITIVA al tercer día de ingreso la recién nacida, por valoración capurro se queda con 34 semanas de edad gestacional, no llora ni respira dando una calificación de Apgar de 5 al primer minuto y 7 a los 5 minutos; por lo que se tuvo que dar dos ciclos de presión positiva sin obtener esfuerzo respiratorio se decide intubación orotraqueal administrando por esta vía una dosis de surfactante exógeno, así como la colocación de acceso venoso umbilical, a la somatometría obtuvo un peso de $1.79 \mathrm{gr}$, talla de $49 \mathrm{~cm}$, perímetro cefálico de $31 \mathrm{~cm}$. Hasta este momento con los diagnósticos de ingreso: Recién Nacido Pretérmino (RNP) de 34 semanas, Síndrome de Dificultad Respiratoria, Depresión por anestésicos, probable Sepsis Neonatal por ruptura prematura de membranas de larga evolución, Sospecha de Infección por SARS-COV 2.

La RNP se ingresa al área de Unidad de Cuidados Intensivos Neonatales bajo técnica de aislamiento, a las 12 horas de ingreso se extuba de manera electiva sin incidentes dejando oxígeno indirecto, con gasometría de control pos extubación en equilibrio acido-base y control radiográfico de tórax observando 9 espacios intercostales, infiltrado parahiliar bilateral sin alguna otra alteración. Pasado el primer día de vida extra uterina comienza la alimentación debido al antecedente de hipoxia al momento del nacimiento, se realiza la extracción del primer control de laboratorios (biometría hemática) resultados alterados con presencia de trombocitopenia y bilirrubinas elevadas. Se le inició fototerapia continua por 72 horas, además de tomar RT-PCR COVID-19 el cual se reporta positivo al tercer día de ingreso continuando con manejo establecido, manifestando evolución insidiosa con distensión abdominal y náuseas, la radiografía de abdomen con datos de gastromegalia y distensión del asa intestinal por lo que se deja en ayuno. Se decide tomar un nuevo muestreo de laboratorios resultados arrojaron alteraciones como disminución del número de leucocitos en sangre, disminución de granulocitos en sangre y nivel bajo de plaquetas, se decide iniciar tratamiento a base de un esquema antimicrobiano (amikacina-ampicilina). Al séptimo día de vida se reinicia la alimentación, así como ultrasonido transfontanelar sin datos de hemorragia, a nivel respiratorio se retira oxígeno indirecto manteniendo saturaciones por arriba del $90 \%$, además de retirar catéter umbilical venoso y colocar Catéter Central de Inserción Periférica (PICC), hemodinámicamente estable.

A los 14 días de vida con evolución favorable cumpliendo 10 días de esquema antimicrobiano y control de laboratorios sin evidencias de respuesta inflamatoria sistémica, adecuada ganancia de peso 1.86 , tolerando la alimentación por técnica mixta no considerando agregar ningún antibiótico.

Se toma nuevo control de RT-PCR a los 20 días de vida (aún positivo) hasta el momento con evolución favorable cumpliendo 36.5 semanas de gestación corregidas y con peso de 1.96 gr, ya con adecuado reflejo de succión y deglución, se pasa a terapia intermedia donde se reporta prueba nuevamente positiva; sin embargo debido a que la evolución fue favorable y la madre evolucionó de igual manera se decide pasar a crecimiento y desarrollo a los 23 días de vida para capacitación de la madre e inicio de lactancia materna segura.

Por consenso con servicio de infectología y epidemiología se considera que el riesgo es mayor al quedarse hospitalizada al no requerir manejo intrahospitalario, considerando además que el aislamiento tanto de la madre como la recién nacida fue de más de 14 días y ambas presentaron evolución favorable se realiza egreso a los 25 días de vida con 38.3 semanas de gestación corregidas y un peso de $2.15 \mathrm{gr}$ brindando indicaciones precisas y de alarma, así como seguimiento.

\section{Discusión.}

Se presenta el caso de un recién nacido de 34 SDG positivo a SARS Cov-2, en el que se describe la evolución clínica desde su ingreso hasta su egreso, la cual es favorable. Las manifestaciones clínicas de la enfermedad causada por el SARS-CoV-2 son inespecíficas: fatiga, cefalea, congestión nasal, rinorrea, tos seca, odinofagia, fiebre y, en los casos graves, disnea e hipoxemia. La neumonía descrita en el curso de la COVID-19 es muy semejante a la neumonía por el SARS y el MERS-CoV. El $81 \%$ de los casos presenta un curso autolimitado de la enfermedad en dos semanas. pacientes que evolucionan hacia formas graves de la enfermedad lo hacen rápidamente a un síndrome de distrés respiratorio agudo (ARDS, por sus siglas en inglés) y a un choque séptico, que eventualmente evoluciona a un fallo múltiple de órganos. (Heng L., 2020) Hay tres series de casos de niños infectados por SARS-CoV-2: la primera incluye 20 niños, hasta el 31 de enero de 2020, en la provincia de Zhejiang; la segunda 34 niños afectados entre el 19 de enero y el 7 de febrero de 2020 en la provincia de Shenzhen y la tercera nueve infantes de diferentes provincias de China. La serie es la que aporta más detalles: ninguno de los pacientes tenía enfermedades de base, el $65 \%$ presentó síntomas respiratorios, el $26 \%$ evolucionó hacia una forma moderada y el $9 \%$ de los niños se encontraban asintomáticos. Los síntomas más comunes fueron fiebre y tos. En esta entre las manifestaciones clínicas más frecuentes se encontraron la ausencia de fiebre, la fiebre baja o moderada, la rinitis, la tos, la fatiga, la cefalea y la diarrea y en los casos más severos, disnea y cianosis; sin embargo, en la serie de nueve casos, solo en cuatro niños se informó fiebre y uno se encontraba asintomático.

Los niños son mucho menos afectados por el SARSCoV-2 que los adultos. El Centro chino para el control y la prevención de enfermedades informó que de 72, 314 enfermos hasta el 11 de febrero de 2020 sólo el 2\% eran menores de 19 años. Las evidencia fisiopatológica, sobre todo en pacientes prematuros es muy inespecífica por lo que requieren una vigilancia estrecha de constantes vitales, la existencia de signos y síntomas patológicos a un nivel respiratorio y 
gastrointestinal, taquipnea, quejido respiratorio, apnea, tos, taquicardia, vómito, fiebre y diarrea así como trombocitopenia, linfopenia, leucopenia, alteraciones en la función hepática y renal. Por lo tanto el paciente hijo de madre positiva a covid-19 sintomático, requiere seguimiento y vigilancia en la UCIN. ${ }^{9}$

Se sugiere evitar el seguimiento innecesario de los recién nacidos; no se recomienda citar a todos los pacientes a la semana de vida sino, de preferencia, informar a todos los padres de las posibles complicaciones que pudieran sobrevenir. Es necesario insistir en la hiperbilirrubinemia. Se sugiere el uso de recursos en línea, videollamadas y aplicaciones para mantener la comunicación directa con el familiar y la vigilancia permanente; además de informar a las familias acerca de COVID-19 y las medidas de prevención necesarias. Para la estancia en su domicilio es importante mencionar a los familiares que limiten el contacto del neonato con adultos senescentes o personas con enfermedades crónicas degenerativas. ${ }^{10}$

Podemos concluir que es fundamental y crucial conocer a fondo la conducción óptima en el paciente neonatal diagnosticado con SARS CoV-2. En la actualidad con más de 6 millones de casos de COVID-19 en todo el mundo, la proporción de neonatos con enfermedad es aún baja. 11 Además, el COVID-19 neonatal parece adquirirse después del nacimiento, aunque no se descarta la transmisión vertical. Este estudio descriptivo demuestra que el seguir los protocolos actuales y evidencia científica impacta de manera positiva ya que la estancia hospitalaria y complicaciones tienden a disminuir.

Conflicto de intereses. Los autores declaran que no tienen conflictos de intereses en el desarrollo de este estudio de caso, cuyo único propósito es académico.

\section{Referencias}

[1] Arturo Cardona Pérez Director General Manuel Cortés Bonilla Director Médico J, Myrna Souraye Godines Enriquez D, Irma Alejandra Coronado Zarco D, Roberto Rodríguez Bosch Subdirector de Obstetricia Dra Rosa Gabriela Hernández Cruz M, Hugo Ramírez Santes V, Ma Isabel Villegas Mota D, et al. Lineamientos COVID-19 INPER. Pub Inper [Internet]. 2020;1(5-80).

[2] Durán, M., Rodrigues, A.. Precauciones en la atención neonatal en la sala de partos ante una madre sospechosa o positiva para COVID-19. Acta Pediatr Mex., 2020;41(Supl 1):S94-S100.

[3] Shaoshuai, W., Lili, G., Ling, C., Weiyong, L., Yong, C., Jingyi, Z., \& Ling, F. (2020). A case report of neonatal COVID-19 infection in China. Clinical infectious diseases: an official publication of the Infectious Diseases Society of America.

[4] Zhu N, Zhang D, Wang W, Li X, Yang B, Song J, et al. A novel coronavirus from patients with pneumonia in China, 2019. N Engl J Med. 2020;382(8):727-33. doi: 10.1056/NEJMoa2001017.

[5] Li, N., Han, L., Peng, M., Lv, Y., Ouyang, Y., Liu, K., ... \& Yang, L. 2020. Maternal and neonatal outcomes of pregnant women with COVID-19 pneumonia: a case-control study. Clinical infectious diseases.
[6] Cai J, Xu J, Lin D, Yang Z, Xu L, Qu Z et al. Case Series of children with 2019 novel coronavirus infection: clinical and epidemiological features. Clin Infect Dis. 2020. PMC7108143. https://doi.org/10.1093/cid/ciaa198.

[7] Hasan A, Mehmood N, Fergie J. Coronavirus Disease (COVID-19) and Pediatric Patients: A Review of Epidemiology, Symptomatology, Laboratory and Imaging Results to Guide the Development of a Management Algorithm. Cureus. 2020; 12(3): e7485.

[8] Ministerio de Salud Costa Rica. Caja Costarricense Seguro Social. Lineamientos generales sobre la lactancia materna y COVID-19. Versión 1.

[9] Macías-Avilés HA. Manejo del neonato sospechoso e infectado de COVID-19 en la UCIN. Acta Pediatr Méx 2020; 41 (Supl 1):S101S108

[10] Durán-Colosio MP, Rodríguez-Weber MA. Precauciones en la atención neonatal en la sala de partos ante una madre sospechosa o positiva para COVID-19. Acta Pediatr Méx 2020; 41 (Supl 1):S94S100

[11] Zimmermann $\mathrm{P}$ and Curtis N. Coronavirus Infections in Children Including COVID-19. An Overview of the Epidemiology, Clinical Features, Diagnosis, Treatment and Prevention Options in Children. Pediatr Infect Dis J 2020; 39:355-368 DOI: 10.1097/INF.0000000000002660 